\title{
Synthesis, spectral characterization and antihaemostatic activity of 1,2,4-triazoles incorporating 1,2,4-triazine rings
}

\author{
RAVINDRA R KAMBLE* and BELGUR S SUDHA \\ Department of Chemistry and Food Science, Yuvaraja's College, University of Mysore, Mysore 570005 \\ e-mail: ravichem1234@ rediffmail.com
}

MS received 16 June 2005; revised 2 January 2006

\begin{abstract}
A simple and high yielding method for the integration of a 1,2,4-triazole ring with 1,2,4-triazine-5-one $(\mathbf{4 a}-\mathbf{j})$ has been developed starting from 3-arylsydnones $(\mathbf{1 a - d})$. The structures were proved by their spectral data and screened for antihaemostatic activity.
\end{abstract}

Keywords. Sydnones; 1,3-dipolar cycloaddition; cyclocondensation; N-N-biheterocycles; antihaemostatic activity.

\section{Introduction}

Sydnones are some of the few heterocycles that have gained importance recently as they readily undergo ring transformation to various heterocycles by $1,3-$ dipolar cycloaddition reaction. The 3-arylsydnones not only offer interesting chemistry but their derivatives possess diverse chemotherapeutic properties. ${ }^{1,2}$ A number of heterocyclic compounds have been synthesized from 3-arylsydnones. ${ }^{3-5}$

The aim of our present work is to extend the 1,3dipolar cycloaddition to synthesise bisheterocycles containing 1,2,4-triazole and 1,2,4-triazine ring systems. 1,2,4-Triazoles have been reported as potential biologically active agents. ${ }^{6-10}$ Triazines find brightening and fibre finishing uses in the textile industry. Triazine derivatives have also been used as chain lengthening agents in polyurethane polymerisation, azodyes, paints, plastic, rubber and also used as fungicides and insecticides. ${ }^{11}$ Zhang et $a l^{12}$ have reported the synthesis and antibacterial activity of 4-aryl-3-(1-p-chloro-phenyl-5-methyl-1,2,3-triazol-4yl)-1,2,4-triazolin-5-thiones.

Prompted by these observations, the N-N biheterocycles, viz. 6-methyl-4-(3'-methyl-5'-oxo-1'-aryl1,5-dihydro-1,2,4-triazol-4'-yl)-3-oxo/thioxo-3,4-dihydro-2H-1,2,4-triazin-5-ones $(\mathbf{4 a - j})$ have been synthesized.

Normally the haemostatic process plays a delicate balance between keeping blood in the fluid state to

*For correspondence maintain flow and rapidly forming an occluding plug following vessel injury. Thrombosis occurs because of alteration in this balance. The recent advances in understanding of the haemostatic process have led to design novel antihaemostatic drugs. In the light of this observation, the title compounds were subjected to preliminary antihaemostatic activity.

\section{Experimental}

\subsection{Materials, methods and instruments}

Melting points were determined in open capillaries and are uncorrected. IR (KBr) spectra were recorded on Nicolet Impact-410 FT-IR spectrophotometer, NMR spectra $\left(\mathrm{CDCl}_{3}\right)$ on Varian-300 MHz FT-NMR spectrometer with TMS as internal standard. Purity of the compounds was checked by TLC. Compounds 1a-d were prepared according to the literature method. ${ }^{2}$

2.2a 6-Methyl-4-(3'-methyl-5'-oxo-1'-phenyl-1,5-dihydro-1,2,4-triazol-4'-yl)-3-oxo-3,4-dihydro-2H-1,2,4-

triazin-5-one (4a): A mixture of $\mathbf{2 a}(10 \mathrm{mmol})$ and semicarbohydrazide (3k, $10 \mathrm{mmol})$ in ethanol $(10 \mathrm{ml})$ was refluxed for about $5 \mathrm{~h}$. Pyruvic acid $(10 \mathrm{mmol})$ and glacial acetic acid $(20 \mathrm{ml})$ fused with sodium acetate $(2 \mathrm{~g})$ were added and further refluxed for $5 \mathrm{~h}$. The reaction mixture was cooled and poured into ice. The solid obtained was filtered off and recrystallized from ethanol gave colourless needles $(2 \cdot 25 \mathrm{~g}, 75 \%)$ of $\mathbf{4 a}$ (cf. table 1$)$. 
Table 1. Characterization data of title compounds $\mathbf{4 a - j}$.

\begin{tabular}{|c|c|c|c|c|c|c|}
\hline \multirow[b]{2}{*}{ Compound } & \multirow[b]{2}{*}{ Molecular formula } & \multirow[b]{2}{*}{ Yield $(\%)$} & \multirow[b]{2}{*}{ m.p. $\left({ }^{\circ} \mathrm{C}\right)$} & \multicolumn{3}{|c|}{ Calculated (found) } \\
\hline & & & & $\mathrm{C}$ & $\mathrm{H}$ & $\mathrm{N}$ \\
\hline $4 a$ & $\mathrm{C}_{13} \mathrm{H}_{12} \mathrm{~N}_{6} \mathrm{O}_{3}$ & 75 & $174-5$ & $52.00(51.96)$ & $4 \cdot 03(4 \cdot 00)$ & $27.99(28.01)$ \\
\hline $4 b$ & $\mathrm{C}_{14} \mathrm{H}_{14} \mathrm{~N}_{6} \mathrm{O}_{3}$ & 77 & $143-4$ & $53 \cdot 50(53.49)$ & $4.49(4.52)$ & $26 \cdot 74(26 \cdot 75)$ \\
\hline $4 c$ & $\mathrm{C}_{14} \mathrm{H}_{14} \mathrm{~N}_{6} \mathrm{O}_{4}$ & 84 & $201-2$ & $50 \cdot 91(50.93)$ & $4 \cdot 27(4 \cdot 25)$ & $25 \cdot 44(25 \cdot 42)$ \\
\hline 4d & $\mathrm{C}_{13} \mathrm{H}_{11} \mathrm{~N}_{6} \mathrm{ClO}_{3}$ & 80 & $165-5$ & $46.65(46.61)$ & $3 \cdot 31(3 \cdot 32)$ & $25 \cdot 11(25 \cdot 08)$ \\
\hline $4 e$ & $\mathrm{C}_{13} \mathrm{H}_{11} \mathrm{~N}_{6} \mathrm{BrO}_{3}$ & 81 & $138-9$ & $41 \cdot 18(41.21)$ & $2.92(2.89)$ & $22 \cdot 16(22 \cdot 18)$ \\
\hline $4 f$ & $\mathrm{C}_{13} \mathrm{H}_{12} \mathrm{~N}_{6} \mathrm{O}_{2} \mathrm{~S}$ & 79 & $192-3$ & $49 \cdot 36(49.34)$ & $3.82(3.79)$ & $26 \cdot 57(26 \cdot 60)$ \\
\hline $4 g$ & $\mathrm{C}_{14} \mathrm{H}_{14} \mathrm{~N}_{6} \mathrm{O}_{2} \mathrm{~S}$ & 85 & $154-5$ & $50.90(50.87)$ & $4 \cdot 27(4 \cdot 23)$ & $25.44(25.41)$ \\
\hline $4 \mathrm{~h}$ & $\mathrm{C}_{14} \mathrm{H}_{14} \mathrm{~N}_{6} \mathrm{O}_{3} \mathrm{~S}$ & 88 & $226-7$ & $48 \cdot 55(48.59)$ & $4.07(4.02)$ & $24 \cdot 26(24 \cdot 28)$ \\
\hline $4 \mathbf{i}$ & $\mathrm{C}_{13} \mathrm{H}_{11} \mathrm{~N}_{6} \mathrm{ClO}_{2} \mathrm{~S}$ & 82 & $134-5$ & $44 \cdot 51(44.48)$ & $3 \cdot 16(3 \cdot 14)$ & $23.96(23.95)$ \\
\hline $4 \mathbf{j}$ & $\mathrm{C}_{13} \mathrm{H}_{11} \mathrm{~N}_{6} \mathrm{BrO}_{2} \mathrm{~S}$ & 80 & $218-9$ & $39.51(39.50)$ & $2 \cdot 81(2 \cdot 83)$ & $21 \cdot 26(21 \cdot 24)$ \\
\hline
\end{tabular}

IR $v_{\max }(\mathrm{KBr}): 1559,1585,1654,1673,3196 \mathrm{~cm}^{-1}$.

${ }^{1} \mathrm{H}$ NMR $\left(\mathrm{CDCl}_{3}\right): \delta 1.98\left(3 \mathrm{H}, s, \mathrm{C}_{6}-\mathrm{CH}_{3}\right), 2.04$ $\left(3 \mathrm{H}, s, \mathrm{C}_{3^{\prime}}-\mathrm{CH}_{3}\right), 6 \cdot 50-6 \cdot 75(5 \mathrm{H}, m, \mathrm{ArH}), 9 \cdot 0(1 \mathrm{H}$, $b s, \mathrm{NH})$.

${ }^{13} \mathrm{C}$ NMR $\left(\mathrm{CDCl}_{3}\right): \delta 16 \cdot 4\left(\mathrm{C}_{6}-\underline{\mathrm{CH}}_{3}\right), 18 \cdot 0\left(\mathrm{C}_{3^{\prime}}{ }^{-}\right.$ $\left.\underline{\mathrm{CH}}_{3}\right), 119 \cdot 5-135 \cdot 6(\mathrm{Ar} \underline{\mathrm{C}}), 151.0\left(\mathrm{C}_{5^{\prime}}\right), 153.0\left(\mathrm{C}_{6}\right)$, $155 \cdot 0\left(\mathrm{C}_{3^{\prime}}\right), 157 \cdot 0\left(\mathrm{C}_{3}\right), 160 \cdot 0\left(\mathrm{C}_{5}\right)$.

$2.2 \mathrm{~b}$ 6-Methyl-4-(3'-methyl-5'-oxo-1'-p-tolyl-1,5-dihydro-1,2,4-triazol-4'-yl)-3-oxo-3,4-dihydro-2H-1,2,4 triazin-5-one (4b): IR $v_{\max }(\mathrm{KBr}): 1544,1585$, $1640,1668,3204 \mathrm{~cm}^{-1}$.

${ }^{1} \mathrm{H}$ NMR $\left(\mathrm{CDCl}_{3}\right): \delta 2 \cdot 12\left(3 \mathrm{H}, s, \mathrm{C}_{6}-\mathrm{CH}_{3}\right), 2 \cdot 20$ $\left(3 \mathrm{H}, s, \mathrm{C}_{3^{\prime}}-\mathrm{CH}_{3}\right), 2.28\left(3 \mathrm{H}, s, \mathrm{ArCH}_{3}\right), 6 \cdot 50(2 \mathrm{H}, d$, $J=8.6 \mathrm{~Hz}, \mathrm{ArH}), 6.74(2 \mathrm{H}, d, J=8.6 \mathrm{~Hz}, \mathrm{ArH})$, $10 \cdot 05(b s, 1 \mathrm{H}, \mathrm{NH})$.

${ }^{13} \mathrm{C}$ NMR $\left(\mathrm{CDCl}_{3}\right): \delta 15.9\left(\mathrm{C}_{6}-\mathrm{CH}_{3}\right), 17.2\left(\mathrm{C}_{3^{\prime}}\right.$ $\left.\mathrm{CH}_{3}\right), 117 \cdot 4-140.6(\mathrm{ArC}), 147 \cdot 0\left(\mathrm{C}_{5^{\prime}}\right), 149 \cdot 0\left(\mathrm{C}_{6}\right)$, $152 \cdot 0\left(\mathrm{C}_{3^{\prime}}\right), 155 \cdot 0\left(\mathrm{C}_{3}\right), 162 \cdot 0\left(\mathrm{C}_{5}\right)$.

2.2c 6-Methyl-4-(3'-methyl-5'-oxo-1'-p-anisyl-1,5-dihydro-1,2,4-triazol-4'-yl)-3-oxo-3,4-dihydro-2H-1,2,4 triazin-5-one (4c): IR $v_{\max }(\mathrm{KBr}): 1560,1595,1625$, $1646,3370 \mathrm{~cm}^{-1}$.

${ }^{1} \mathrm{H}$ NMR $\left(\mathrm{CDCl}_{3}\right): \delta 2 \cdot 10\left(3 \mathrm{H}, s, \mathrm{C}_{6}-\mathrm{CH}_{3}\right), 2 \cdot 15$ $\left(3 \mathrm{H}, s, \mathrm{C}_{3^{\prime}}-\mathrm{CH}_{3}\right), 3.50\left(3 \mathrm{H}, s, \mathrm{OCH}_{3}\right), 6.77(2 \mathrm{H}, d$, $J=5.9 \mathrm{~Hz}, \mathrm{ArH}), 6.95(2 \mathrm{H}, d, J=5.9 \mathrm{~Hz}, \mathrm{ArH}), 9.8$ (bs, 1H, NH).

${ }^{13} \mathrm{C}$ NMR $\left(\mathrm{CDCl}_{3}\right): \delta 15.9\left(\mathrm{C}_{6}-\mathrm{C}_{3}\right), 17 \cdot 2.0\left(\mathrm{C}_{3^{\prime}}-\right.$ $\left.\underline{\mathrm{CH}}_{3}\right), 50 \cdot 7\left(\mathrm{OCH}_{3}\right), 117 \cdot 4-140 \cdot 6(\mathrm{ArC}), 147 \cdot 0\left(\mathrm{C}_{5^{\prime}}\right)$, $149 \cdot 0\left(\mathrm{C}_{6}\right), 152 \cdot 0\left(\mathrm{C}_{3^{\prime}}\right), 155 \cdot 0\left(\mathrm{C}_{3}\right), 162 \cdot 0\left(\mathrm{C}_{5}\right)$.

2.2d 6-Methyl-4-(3'-methyl-5'-oxo-1'-p-chlorophenyl1,5-dihydro-1,2,4-triazol-4'-yl)- 3-oxo-3,4-dihydro2H-1,2,4 triazin-5-one $(4 \mathrm{~d}): \quad \mathrm{IR} \mathrm{v}_{\max }(\mathrm{KBr}): 1560$, 1600, 1610, 1667, $3185 \mathrm{~cm}^{-1}$.

${ }^{1} \mathrm{H}$ NMR $\left(\mathrm{CDCl}_{3}\right): \delta 2 \cdot 25\left(3 \mathrm{H}, s, \mathrm{C}_{6}-\mathrm{CH}_{3}\right), 2 \cdot 34$ $\left(3 \mathrm{H}, s, \mathrm{C}_{3^{\prime}}-\mathrm{CH}_{3}\right), 6 \cdot 85(2 \mathrm{H}, d, J=9 \cdot 1 \mathrm{~Hz}, \mathrm{ArH}), 7 \cdot 01$ $(2 \mathrm{H}, d, J=9 \cdot 1 \mathrm{~Hz}, \mathrm{ArH}), 11 \cdot 2(b s, 1 \mathrm{H}, \mathrm{NH})$.
${ }^{13} \mathrm{C}$ NMR $\left(\mathrm{CDCl}_{3}\right): \delta 16 \cdot 0\left(\mathrm{C}_{6}-\underline{\mathrm{CH}}_{3}\right), 17 \cdot 8\left(\mathrm{C}_{3^{\prime}-}\right.$ $\left.\underline{\mathrm{CH}}_{3}\right), 119 \cdot 2-139.5(\mathrm{ArC}), 150 \cdot 0\left(\mathrm{C}_{5^{\prime}}\right), 151.9\left(\mathrm{C}_{6}\right)$, $154 \cdot 0\left(\mathrm{C}_{3^{\prime}}\right), 158 \cdot 0\left(\mathrm{C}_{3}\right), 165 \cdot 0\left(\mathrm{C}_{5}\right)$.

2.2e 6-Methyl-4-(3'-methyl-5'-oxo-1'-p-bromophenyl1,5-dihydro-1,2,4-triazol-4'-yl)- 3-oxo-3,4-dihydro2H-1,2,4 triazin-5-one (4e): IR $v_{\max }(\mathrm{KBr}): 1574$, $1598,1625,1680,3320 \mathrm{~cm}^{-1}$.

${ }^{1} \mathrm{H}$ NMR $\left(\mathrm{CDCl}_{3}\right): \delta 2.40\left(3 \mathrm{H}, s, \mathrm{C}_{6}-\mathrm{CH}_{3}\right), 2.58$ $\left(3 \mathrm{H}, s, \mathrm{C}_{3^{\prime}}-\mathrm{CH}_{3}\right), 7 \cdot 12(2 \mathrm{H}, d, J=6.8 \mathrm{~Hz}, \mathrm{ArH}), 7.25$ $(2 \mathrm{H}, d, J=6.9 \mathrm{~Hz}, \mathrm{ArH}), 11.0(b s, 1 \mathrm{H}, \mathrm{NH})$.

${ }^{13} \mathrm{C}$ NMR $\left(\mathrm{CDCl}_{3}\right): \delta 17.5\left(\mathrm{C}_{6}-\mathrm{CH}_{3}\right), 19.5\left(\mathrm{C}_{3^{\prime}}-\right.$ $\left.\mathrm{CH}_{3}\right), 118.7-137.4(\mathrm{ArC}), 152.0 \quad\left(\mathrm{C}_{5^{\prime}}\right), 154\left(\mathrm{C}_{6}\right)$, $155 \cdot 0\left(\mathrm{C}_{3^{\prime}}\right), 157 \cdot 0\left(\mathrm{C}_{3}\right), 168 \cdot 0\left(\mathrm{C}_{5}\right)$.

$2.3 \mathrm{f}$ 6-Methyl-4-(3'-methyl-5'-oxo-1'-phenyl-1,5-dihydro-1,2,4-triazol-4'-yl)-3-thioxo-3,4-dihydro-2H-1,2,4triazin-5-one (4f): The compound $\mathbf{4 f}$ was prepared by the reaction of $\mathbf{2 a}$ with thiosemicarbohydrazides 31 followed by the reaction with pyruvic acid as explained in the experimental for $\mathbf{4 a}$ (cf. table 1).

IR $v_{\max }(\mathrm{KBr}): 1310,1565,1591,1620,3295 \mathrm{~cm}^{-1}$.

${ }^{1} \mathrm{H}$ NMR $\left(\mathrm{CDCl}_{3}\right): \delta 1.95\left(3 \mathrm{H}, s, \mathrm{C}_{6}-\mathrm{CH}_{3}\right), 2.04$ $\left(3 \mathrm{H}, s, \mathrm{C}_{3^{\prime}}-\mathrm{CH}_{3}\right), 7 \cdot 00-7.64(5 \mathrm{H}, m, \mathrm{ArH}), 11.70$ $(1 \mathrm{H}, b s, \mathrm{NH})$.

${ }^{13} \mathrm{C}$ NMR $\left(\mathrm{CDCl}_{3}\right): \delta 15.8\left(\mathrm{C}_{6}-\mathrm{C}_{3}\right), 17 \cdot 4\left(\mathrm{C}_{3^{\prime}}\right.$ $\left.\mathrm{CH}_{3}\right), 120 \cdot 4-138 \cdot 2(\mathrm{ArC}), 150 \cdot 0\left(\mathrm{C}_{5^{\prime}}\right), 152 \cdot 0\left(\mathrm{C}_{6}\right)$, $155 \cdot 0\left(\mathrm{C}_{3^{\prime}}\right), 163 \cdot 0\left(\mathrm{C}_{5}\right), 183 \cdot 0\left(\mathrm{C}_{3}\right)$.

$2.3 \mathrm{~g}$ 6-Methyl-4-(3'-methyl-5'-oxo-1'-p-tolyl-1,5-dihydro-1,2,4-triazol-4'-yl)-3-thioxo-3,4-dihydro-2H-1,2,4triazin-5-one $(\mathbf{4 g})$ : IR $v_{\max }(\mathrm{KBr}): 1315,1570,1615$, $1667,3250 \mathrm{~cm}^{-1}$.

${ }^{1} \mathrm{H}$ NMR $\left(\mathrm{CDCl}_{3}\right): \delta 2.00\left(3 \mathrm{H}, s, \mathrm{C}_{6}-\mathrm{CH}_{3}\right), 2 \cdot 10$ $\left(3 \mathrm{H}, s, \mathrm{C}_{3^{\prime}}-\mathrm{CH}_{3}\right), 2.35\left(3 \mathrm{H}, s, \mathrm{ArCH}_{3}\right), 7 \cdot 04(2 \mathrm{H}, d$, $J=6.9 \mathrm{~Hz}, \mathrm{ArH}), 7.15(2 \mathrm{H}, d, J=6.9 \mathrm{~Hz}, \mathrm{ArH}), 9.7$ $(1 \mathrm{H}, b s, \mathrm{NH})$.

${ }^{13} \mathrm{C}$ NMR $\left(\mathrm{CDCl}_{3}\right): \delta 15.8\left(\mathrm{C}_{6}-\underline{\mathrm{CH}}_{3}\right), 17 \cdot 4\left(\mathrm{C}_{3^{\prime}}\right.$ $\left.\underline{\mathrm{CH}}_{3}\right), 120 \cdot 4-138 \cdot 2(\mathrm{ArC}), 150 \cdot 0\left(\mathrm{C}_{5^{\prime}}\right), 152 \cdot 0\left(\mathrm{C}_{6}\right)$, $155 \cdot 0\left(\mathrm{C}_{3^{\prime}}\right), 163 \cdot 0\left(\mathrm{C}_{5}\right), 183 \cdot 0\left(\mathrm{C}_{3}\right)$. 

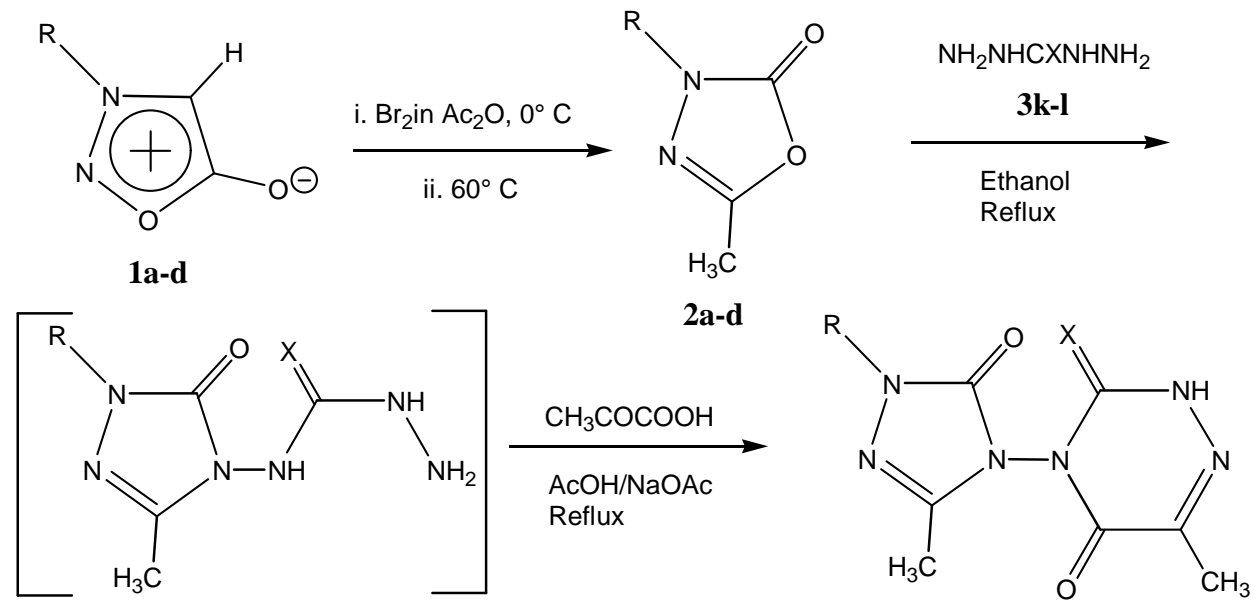

4a-j

$\mathbf{a} ; \mathrm{R}=\mathrm{C}_{6} \mathrm{H}_{5}, \mathrm{X}=\mathrm{O}, \mathbf{b} ; \mathrm{R}=p-\mathrm{CH}_{3} \mathrm{C}_{6} \mathrm{H}_{4}, \mathrm{X}=\mathrm{O}, \mathbf{c} ; \mathrm{R}=p-\mathrm{OCH}_{3} \mathrm{C}_{6} \mathrm{H}_{4}, \mathrm{X}=\mathrm{O}, \mathbf{d} ; \mathrm{R}=p-\mathrm{ClC}_{6} \mathrm{H}_{4}$, $\mathrm{X}=\mathrm{O}, \mathbf{e} ; \mathrm{R}=p-\mathrm{BrC}_{6} \mathrm{H}_{4}, \mathrm{X}=\mathrm{O}, \mathbf{f} ; \mathrm{R}=\mathrm{C}_{6} \mathrm{H}_{5}, \mathrm{X}=\mathrm{S}, \mathbf{g} ; \mathrm{R}=p-\mathrm{CH}_{3} \mathrm{C}_{6} \mathrm{H}_{4}, \mathrm{X}=\mathrm{S}, \mathbf{h} ; \mathrm{R}=$

$p-\mathrm{OCH}_{3} \mathrm{C}_{6} \mathrm{H}_{4}, \mathrm{X}=\mathrm{S}, \mathbf{i} ; \mathrm{R}=p-\mathrm{ClC}_{6} \mathrm{H}_{4}, \mathrm{X}=\mathrm{S}, \mathbf{j} ; \mathrm{R}=p-\mathrm{BrC}_{6} \mathrm{H}_{4}, \mathrm{X}=\mathrm{S}, \mathbf{k} ; \mathrm{X}=\mathrm{O}, \mathbf{l} ; \mathrm{X}=\mathrm{S}$.

Scheme 1.

$2.3 \mathrm{~h}$ 6-Methyl-4-(3'-methyl-5'-oxo-1'-p-anisyl-1,5dihydro-1,2,4-triazol-4'-yl)-3-thioxo-3,4-dihydro-2H1,2,4-triazin-5-one (4h): $\quad \mathrm{IR} \mathrm{v}_{\max }(\mathrm{KBr}): 1318,1550$, $1605,1631,3390 \mathrm{~cm}^{-1}$.

${ }^{1} \mathrm{H}$ NMR $\left(\mathrm{CDCl}_{3}\right): \delta 2 \cdot 01\left(3 \mathrm{H}, s, \mathrm{C}_{6}-\mathrm{CH}_{3}\right), 2 \cdot 12$ $\left(3 \mathrm{H}, s, \mathrm{C}_{3^{\prime}}-\mathrm{CH}_{3}\right), 3.73\left(3 \mathrm{H}, s, \mathrm{OCH}_{3}\right), 6.75(2 \mathrm{H}, d$, $J=7.4 \mathrm{~Hz}, \mathrm{ArH}), 7.03(2 \mathrm{H}, d, J=7.4 \mathrm{~Hz}, \mathrm{ArH})$, $12 \cdot 0(1 \mathrm{H}, b s, \mathrm{NH})$.

${ }^{13} \mathrm{C}$ NMR $\left(\mathrm{CDCl}_{3}\right): \delta 16 \cdot 0\left(\mathrm{C}_{6}-\mathrm{C}_{3}\right), 19 \cdot 0\left(\mathrm{C}_{3^{\prime}}\right.$ $\left.\mathrm{CH}_{3}\right), 56 \cdot 0\left(\mathrm{OCH}_{3}\right), 114 \cdot 3-140 \cdot 6(\mathrm{ArC}), 144 \cdot 0\left(\mathrm{C}_{5^{\prime}}\right)$, $149 \cdot 0\left(\mathrm{C}_{6}\right), 151 \cdot 0\left(\mathrm{C}_{3^{\prime}}\right), 160 \cdot 0\left(\mathrm{C}_{5}\right), 181 \cdot 0\left(\mathrm{C}_{3}\right)$.

$2.3 \mathrm{i}$ 6-Methyl-4-(3'-methyl-5'-oxo-1'-p-chlorophenyl1,5-dihydro-1,2,4-triazol-4'-yl)-3-thioxo-3,4-dihydro2H-1,2,4-triazin-5-one (4i): IR $v_{\max }(\mathrm{KBr}): 1305$, $1572,1600,1645,3199 \mathrm{~cm}^{-1}$.

${ }^{1} \mathrm{H}$ NMR $\left(\mathrm{CDCl}_{3}\right): \delta 2 \cdot 12\left(3 \mathrm{H}, s, \mathrm{C}_{6}-\mathrm{CH}_{3}\right), 2 \cdot 34$ $\left(3 \mathrm{H}, s, \mathrm{C}_{3^{\prime}}-\mathrm{CH}_{3}\right), 7 \cdot 31(2 \mathrm{H}, d, J=5 \cdot 2 \mathrm{~Hz}, \mathrm{ArH}), 7 \cdot 49$ $(2 \mathrm{H}, d, J=5.2 \mathrm{~Hz}, \mathrm{ArH}), 11.00(1 \mathrm{H}, b s, \mathrm{NH})$.

${ }^{13} \mathrm{C}$ NMR $\left(\mathrm{CDCl}_{3}\right): \delta 16.9\left(\mathrm{C}_{6}-\mathrm{CH}_{3}\right), 20 \cdot 5\left(\mathrm{C}_{3^{\prime}}-\right.$ $\left.\mathrm{CH}_{3}\right), 121 \cdot 8-136 \cdot 3(\mathrm{ArC}), 151 \cdot 0\left(\mathrm{C}_{5^{\prime}}\right), 153 \cdot 0\left(\mathrm{C}_{6}\right)$, $157 \cdot 0\left(\mathrm{C}_{3^{\prime}}\right), 164 \cdot 0\left(\mathrm{C}_{5}\right), 186 \cdot 0\left(\mathrm{C}_{3}\right)$.

$2.3 \mathrm{j}$ 6-Methyl-4-(3'-methyl-5'-oxo-1'-p-bromophenyl1,5-dihydro-1,2,4-triazol-4'-yl)-3-thioxo-3,4-dihydro2H-1,2,4-triazin-5-one (4j): IR $v_{\max }(\mathrm{KBr}): 1275$, $1580,1612,1640,3401 \mathrm{~cm}^{-1}$.

${ }^{1} \mathrm{H}$ NMR $\left(\mathrm{CDCl}_{3}\right): \delta 2 \cdot 19\left(3 \mathrm{H}, s, \mathrm{C}_{6}-\mathrm{CH}_{3}\right), 2 \cdot 35$ $\left(3 \mathrm{H}, s, \mathrm{C}_{3^{\prime}}-\mathrm{CH}_{3}\right), 7.26(2 \mathrm{H}, d, \mathrm{ArH}, J=8.0 \mathrm{~Hz}), 7.40$ $(2 \mathrm{H}, d, J=8.0 \mathrm{~Hz}, \mathrm{ArH}), 11.75(1 \mathrm{H}, b s, \mathrm{NH})$.
${ }^{13} \mathrm{C}$ NMR $\left(\mathrm{CDCl}_{3}\right): \delta 19.5\left(\mathrm{C}_{6}-\mathrm{CH}_{3}\right), 21.0\left(\mathrm{C}_{3^{\prime}}\right.$ $\left.\mathrm{CH}_{3}\right), 124 \cdot 7-140 \cdot 3(\mathrm{ArC}), 154 \cdot 0\left(\mathrm{C}_{5^{\prime}}\right), 156 \cdot 0\left(\mathrm{C}_{6}\right)$, $160 \cdot 0\left(\mathrm{C}_{3^{\prime}}\right), 165 \cdot 0\left(\mathrm{C}_{5}\right), 188 \cdot 0\left(\mathrm{C}_{3}\right)$.

\subsection{Antihaemostatic screening}

Tail bleeding time in conscious mice was used to determine antihaemostatic activity of title compounds. ${ }^{13}$ Mice of either sex weighing 20-25 g were divided into seven groups comprising ten mice in each group. Control group received $0.4 \mathrm{ml}$ of $2 \%$ gum acacia. Test compounds were administered in $2 \%$ gum acacia to the remaining six groups. Thirty minutes after administration of the test compounds tail-bleeding time was measured and the results are shown in table 1.

\section{Results and discussion}

\subsection{Preparation of title compounds}

Synthesis of N-N biheterocycles, which is effected sequentially, requires bifunctional precursors, which are not always readily accessible. the 3-arylsydnone 1a-d served as important synthetic precursor for the synthesis of title compounds. ${ }^{2}$ Compound 1a-d was subjected to bromination at $0^{\circ} \mathrm{C}$ followed by heating at $60^{\circ} \mathrm{C}$ underwent 1,3 -dipolar cycloaddition with acetic anhydride to form 3-aryl-5-methyl-2-oxo- $\Delta^{4}$ - 
1,3,4-oxadiazole (2a-d). When the compound 2a-d was refluxed with $3 \mathbf{k}$ for about $5 \mathrm{~h}$ whereby transient ring opening, insertion of terminal nitrogen gave intermediates which underwent cyclisation in-situ readily with pyruvic acid in presence of acetic acid fused with sodium acetate to 6-methyl-4-(3'-methyl5'-oxo-1'-aryl-1,5-dihydro-1,2,4-triazol-4'-yl)-3-oxo3,4-dihydro-2h-1,2,4-triazin-5-one (4a-e). Similarly, the compound $\mathbf{4 f - j}$ was prepared using $\mathbf{3 l}$ (scheme 1).

The mechanism of the reaction involves the nucleophillic attack of $\mathbf{3}$ to carbonyl carbon atom of 2, which proceeds to the formation of a condensed product that in turn eliminates a molecule of water. The other end of the $\mathbf{3}$ reacts with pyruvic acid followed by intramolecular cyclocondensation gave $\mathrm{N}$ $\mathrm{N}$ bicyclic product $(\mathbf{4 a} \mathbf{a}-\mathbf{j})$.

The IR, ${ }^{1} \mathrm{H}$ and ${ }^{13} \mathrm{C}$ NMR spectra of the title compounds were in complete agreement with the assigned structure. In case of IR spectral analyses a broad band due to $\mathrm{NH}$ group was appeared in all the title compounds between 3185 and $3401 \mathrm{~cm}^{-1}$. The amide carbonyl functions appeared as sharp band in the range $1591-1680 \mathrm{~cm}^{-1}$. Another band around $1275-1315 \mathrm{~cm}^{-1}$ was appeared in the compounds $\mathbf{4 f}-\mathbf{j}$ due to $\mathrm{C}=\mathrm{S}$ group. ${ }^{1} \mathrm{H}$ NMR spectral analyses of the title compounds exhibited two singlets at $\delta 1.95$ $2.58 \mathrm{ppm}$ assigned to two methyl groups on $\mathrm{C}_{6}$ and

Table 2. Results of antihaemostatic activity of the title compounds $\mathbf{4 a - j}$.

\begin{tabular}{lcc}
\hline Compound & $\begin{array}{c}\text { Dose } \\
\left(\mathrm{mg} \mathrm{kg}^{-1}\right)\end{array}$ & $\begin{array}{c}\text { Average bleeding time } \\
\text { (in seconds } \pm \text { SEM) }\end{array}$ \\
\hline $\mathbf{4 a}$ & 100 & $158 \cdot 0 \pm 36 \cdot 5$ \\
$\mathbf{4 b}$ & 100 & $144 \cdot 5 \pm 29 \cdot 6$ \\
$\mathbf{4 c}$ & 100 & $170 \cdot 3 \pm 30 \cdot 5$ \\
$\mathbf{4 d}$ & 100 & $180 \cdot 3 \pm 10 \cdot 3$ \\
$\mathbf{4 e}$ & 100 & $370 \cdot 5 \pm 14 \cdot 6$ \\
$\mathbf{4 f}$ & 100 & $359 \cdot 8 \pm 14 \cdot 3$ \\
$\mathbf{4 g}$ & 100 & $403 \cdot 8 \pm 15 \cdot 6$ \\
$\mathbf{4 h}$ & 100 & $400 \cdot 0 \pm 14 \cdot 3$ \\
$\mathbf{4 i}$ & 100 & $445 \cdot 7 \pm 20 \cdot 5$ \\
$\mathbf{4 j}$ & 100 & $470 \cdot 5 \pm 30 \cdot 2$ \\
$2 \%$ Gum acacia & $0 \cdot 4 \mathrm{ml}$ & $89 \cdot 2 \pm 12 \cdot 8$ \\
$\quad$ (control) & & $491 \cdot 1 \pm 49 \cdot 5$ \\
Indomethacin & 100 & \\
$\quad$ (standard) & & \\
\hline
\end{tabular}

$\mathrm{C}_{3^{\prime}}$ carbons. All the title compounds have shown a broad singlet in the range $\delta 9 \cdot 0-12.0 \mathrm{ppm}$ due to $\mathrm{NH}$. Aromatic protons appeared in the expected range as two doublets $\left(\mathrm{AA}^{\prime} \mathrm{BB}^{\prime}\right) .{ }^{13} \mathrm{C}$ NMR spectral analyses showed a number of signals that are consistent with the number of carbons in the molecule.

\subsection{Antihaemostatic activity}

Antihaemostasis results (table 2) reveal that the final compounds do not show activity more than or equal to standard drug indomethacin. Only compounds containing the $\mathrm{C}=\mathrm{S}$ group with $p$-bromophenyl substituent $\mathbf{4 j}(470 \cdot 5)$ and $p$-chlorophenyl substituent $\mathbf{7 p}$ (445.7) show considerable antihaemostatic activity. The oxo derivative $\mathbf{4 a - e}$ exhibits poor antihaemostasis.

\section{Acknowledgements}

The authors are grateful to Mukthar Ahmed, Karnatak University, Dharwad for antihaemostatic analyses.

\section{References}

1. Earl J C and Mckney A W 1935 J. Chem. Soc. 899

2. Mallur S G and Badami B V 2000 Farmaco 5565

3. Hiremath U S, Yelamaggad C V and Badami B V 1995 Indian J. Heterocycl. Chem. 519

4. Havanur S B and Puranik G S 1985 Indian J. Chem. B24 864

5. Badami B V and Puranik G S 1974 Indian J. Chem. B12 671

6. Hosur M C, Talwar M B, Laddi U V, Bennur R S and Bennur S C 1995 Indian J. Chem. B34 707

7. Hussain M I and Amin M 1986 J. Indian Chem. Soc. 63317

8. Gokce M, Cakir B, Erol K and Sahin M F 2001 Arch. Pharm. 334279

9. Duran A, Dogan H N and Rollas S 2002 Farmaco 57 559

10. Xin-Zhi L I and Zong-Xing S I 2002 Chinese J. Org. Chem. 22178

11. Deohate P P and Berad B N 2005 Indian J. Chem. B44 638

12. Sun X W, Liang H T and Zhang Z Y 1999 Indian J. Chem. B38 679

13. Hornstra G, Christ-Hazelholt E, Haddeman E, Ten H F and Nagsteren D H 1981 Prostaglandins 21727 